\title{
R. Neugart
}

CERN, Geneva, Switzerland

\begin{abstract}
Collinear laser spectroscopy in the fast beams of on-line isotope separators is shown to be a powerful tool for isotope shift and hyperfine structure studies in long isotopic chains. The experimental realization is discussed in some detail, and present results are reviewed, with emphasis on the very recent work at ISOLDE. Examples of information on nuclear behaviour are given.
\end{abstract}

\section{CERN LIBRARIES, GENEVA}

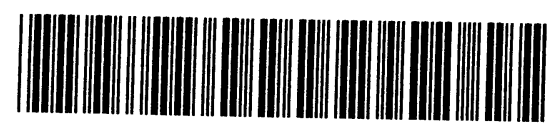

CM-P00071030

Paper presented at the

10th Internat. Conf. on Electromagnetic Isotope Separators and Techniques related to their Applications (EMIS 10) Zinal, Switzerland, 1-6 September 1980

(To appear in Nuclear Instruments and Methods) 


\section{INTRODUCTION}

The past 10 years have seen impressive progress in the field of on-1ine isotope separation. Many new isotopes, far from stability, of an increasing number of elements became accessible for detailed studies of nuclear structure ${ }^{1,2)}$. A considerable part of this work has been due to the outstanding performance of ISOLDE at the CERN $600 \mathrm{MeV}$ Synchro-cyclotron (SC) ${ }^{3)}$. Here, an important role was played by atomic spectroscopy, yielding basic nuclear ground-state properties such as spins, magnetic dipole and electric quadrupole moments, and the variation of the mean-square nuclear charge radius along an isotopic shain. These are obtained from the hyperfine structure (hfs) and isotope shift (IS) in atomic spectra which, about 50 years ago, were amongst the earliest sources of information on nuclear structure ${ }^{4)}$.

Starting with conventional optical spectroscopy, the experimental technique was refined more and more so as to increase the resolution. Radio-frequency methods led to highly precise measurements of atomic ground-state and excitedstate hfs. In the past 10 years, tunable dye lasers came into play, increasing the resolution of optical spectroscopy by several orders of magnitude ${ }^{5}$ ). And by using the methods of Doppler-free spectroscopy, not only could the hfs of hitherto unaccessible states be measured but also the first high-precision measurements of IS were achieved. Because of this work, there is at present a rather complete knowledge of the nuclear parameters for stable and long-lived radioactive nuclides.

The extension to short-lived isotopes far from stability has to face some general problems: i) the small amount of material available in the samples requires ultimate sensitivity; $i i)$ short half-lives require on-line experiments and exclude lengthy procedures of sample preparation; iii) the radioactivity may cause severe background problems, but, on the other hand, it can in some cases be used for efficient detection.

A number of methods could be adapted to this new situation, each of them subject to some applicability restraint due to specific chemical properties and 
energy levels of the element or group of elements concerned. Rather comprehensive work has now been done on mercury and cadmium, the alkali elements and, most recently, on barium. The experimental progress and its essential results have been reviewed by Otten $\left.\left.{ }^{6}, 7\right), \mathrm{K} l u g e^{8}\right)$, and Jacquinot and $\mathrm{Klapisch}^{9}$ ). The 1atest development is included in the very recent conference reports by Otten ${ }^{10}$ ) and Klapisch ${ }^{11}$ ).

\section{THE COLLINEAR-BEAM METHOD}

This paper will deal with the concept of laser spectroscopy with collinear beams. By directly using the fast output beams of isotope separators, this method offers a simple and elegant way of overcoming the problems of sample preparation. Its resolution and sensitivity have been demonstrated to be competitive compared with any other method ${ }^{2}$ ), and it can be expected to be of rather general use.

\subsection{Principle}

This concept is suggested by the finding that the velocity distribution along the fast ion beam corresponds to a Doppler width ideally matching the laser linewidth and the natural width of strong optical lines. Since the energy spread of the ions remains constant during electrostatic acceleration, and

$$
\delta \mathrm{E}=\delta\left(\frac{1}{2} \mathrm{mv}^{2}\right)=\mathrm{mv} \delta \mathrm{v},
$$

the increase of the velocity component $v_{z}$ goes together with a narrowing of the velocity spread $\delta v_{z}$. Thus the Doppler width is narrowed by the same amount inversely proportional to the Doppler shift introduced by acceleration. Using $\mathrm{v}=(2 \mathrm{eU} / \mathrm{m})^{\frac{1}{2}}$ and $\delta \nu_{\mathrm{D}}=v_{0}\left(\delta \mathrm{v}_{\mathrm{z}} / \mathrm{c}\right)$, we obtain

$$
\delta \nu_{D}=v_{0} \frac{\delta E}{\left(2 \mathrm{eU} \mathrm{mc}^{2}\right)^{\frac{1}{2}}} \text {, }
$$

where $\nu_{0}$ is the optical frequency involved in the transition.

Let us take an example. A standard ISOLDE beam has an energy of eU $=60 \mathrm{keV}$, and the energy spread of a thermal surface ionization source can be supposed to be $\delta E=1 \mathrm{eV}$. For a medium-mass element, the absorption of an optical frequency 
of $5 \times 10^{14} \mathrm{~Hz}$ would be subject to a Doppler broadening of $5 \mathrm{MHz}$. For comparison, $10 \mathrm{MHz}$ is the order of magnitude of the natural line-width in strong resonance transitions.

It should be stressed that this high resolution is achieved without loss of particles interacting with the light. Optical resonance cross-sections are of the order $\lambda^{2} \approx 10^{-9} \mathrm{~cm}^{2}$, and with the moderate laser power of a few milliwatts (assuming a beam diameter of $3 \mathrm{~mm}$ ) a transition can be saturated. Correspondingly, each atom is excited many times over a travelling distance of a few centimetres. In most cases, however, optical pumping removes the atoms from the absorbing state, thus limiting the number of possible excitations.

The straightforward way of detection is to observe fluorescence photons. Here the efficiency and background conditions determine essentially the over-all sensitivity, i.e. the minimum flux of particles required for a measurement.

\subsection{Charge-transfer neutralization}

Only in a few cases can the beam of singly charged ions be directly used for spectroscopy with CW dye lasers. Present lasers cover roughly the visible and near infrared spectrum, whereas ionic resonance lines usually lie in the ultraviolet. The range of application of fast-beam spectroscopy can be very much enlarged by converting the ion beam into a neutral atomic beam. Effective conversion is obtained in charge-transfer collisions of the type

$$
\mathrm{B}^{+}+\mathrm{A} \rightarrow \mathrm{B}+\mathrm{A}^{+}+\Delta \mathrm{E}
$$

where $B$ refers to the particles forming the fast beam and A represents an atomic vapour $^{13)}$. Alkali metal vapours are preferred as collision targets because of their high vapour pressure at moderate temperature and their 1oosely bound valence electrons. Cross-sections reach about $10^{-14} \mathrm{~cm}^{2}$ in the quasi-resonant cases where the energy defect $\Delta \mathrm{E}$ is sma11. This corresponds to impact parameters much larger than atomic radii. Consequently, the momentum transfer to the target atom is negligible and the phase-space distribution of the beam is not enlarged. 
Conservation laws require the kinetic energy transferred to the target particle in forward scattering to be of the order $(\Delta E)^{2} / e U$, which is negligible for beam energies in the keV range. For non-resonant charge transfer this means that the energy loss (gain) of the particles in the fast beam equals almost exactly $\Delta \mathrm{E}$.

Following these principles, we are led to a promising scheme of high-resolution spectroscopy for on-line use with radioactive isotope-separated beams: to merge the ion beam with a narrow-band laser beam, convert the ions into atoms, and observe the fluorescence light. This scheme was discussed by Kaufman in $1976{ }^{14}$ ), and a first experimental approach on stable ${ }^{23} \mathrm{Na}$ and ${ }^{133} \mathrm{Cs}$ was reported in $1977 / 78^{15)}$. The pilot work led directly to a series of experiments on unstable isotopes which will be the subject of this report. Independently, Wing et a1. ${ }^{16}$ ) had shown the kinematic compression of Doppler width in the infrared vibrationrotational transitions of the $\mathrm{HD}^{+}$molecular ion. In the following, a number of experiments used the population of metastable states in the ion source for spectroscopic studies of atomic ions, starting with the work of Meier et a1. ${ }^{17}$ ).

\section{EXPERIMENTAL}

\subsection{The apparatus}

The following description of the apparatus refers essentially to the improved set-up used in recent experiments at ISOLDE. Its essential parts are shown in fig. 1. The laser light-source is an actively stabilized commercial singlefrequency dye laser, pumped with an argon ion laser. The mass-separated ion beam is bent into the laser beam by a pair of deflection plates, focused slightly by an electrostatic quadrupole lens, and neutralized in the charge-exchange cell. Excitation of the fast atoms is detected via their fluorescence. The photons emitted along $20 \mathrm{~cm}$ of the beam path are collected by cylindrical optics, focused on the entrance slit of a light-guide, and transmitted to the photomultiplier. Single-photon pulses are counted in the computer CAMAC link. 
For low background, special care must be taken to i) reduce the stray light by an arrangement of sharp-edged black diaphragms at the entrance and exit of the observation chamber; ii) cool the photomultiplier; iii) shield the optical elements and the photomultiplier against radioactivity. The stray light background depends very sensitively on the laser beam shape and the more or less perfect alignment of the apparatus. It has been possible to achieve $10^{3}$ counts/s per mW laser power, but typical running conditions are a factor of 5 or 10 higher. Compared to this, the background from radioactivity and collisional excitation in the rest gas is usually small, except for strong beams where the fluorescence signals are strong as well.

The collection efficiency of the optical system is about $2 \%$ of $4 \pi$, including reflection loss on surfaces and the transmission of the light pipe. With the quantum efficiency of the photomultiplier (depending on the wavelength), a fraction of 2 to $4 \times 10^{-3}$ of the photons emitted by the beam are actually counted.

\subsection{High voltage}

To exploit the full resolution and sensitivity of the method according to Section 2.1, the isotope separator has to be operated with a highly stable acce1eration voltage. The stability requirements for ISOLDE $(U=60 \mathrm{keV})$ are at the $10^{-5}$ level. This has been fulfilled by a series regulation unit ${ }^{18)}$ which receives d.c. and a.c. error signals from ground level via a high-frequency modulated light signal and a capacitor, respectively. The d.c. signal is created by comparing the current through the highly stable measuring voltage divider, with the current of a reference standard. The excellent performance of this device ensures an over-all stability of less than $0.5 \mathrm{~V}$ (maximum deviation over one day), even for high load variations up to $10 \mathrm{~mA}$ due to ionized air created by the proton beam in the target/ion source surroundings.

\subsection{Measuring procedure}

The laser wavelength seen by the fast atoms is conveniently tuned by changing the beam energy. To avoid any interference with the stabilized high-voltage 
supply and the simultaneous change of the separator field and the beam optics, this is accomplished at the measuring site. Therefore, the charge-exchange cell is an integral part of a retardation/acceleration system, designed for a $\pm 10 \mathrm{keV}$ change of the beam energy with a small change of focusing in the interaction region.

In a measurement, the transition frequency(ies) of one isotope have to be compared with a known (stable) reference isotope. For this purpose, beams of different isotopes are alternately directed through the apparatus by changing the separator field in preset steps. Simultaneously, the high voltage is switched between fixed values, roughly set to the expected line positions. A sweep voltage is additionally applied for fine tuning, controlled by the computer which takes the fluorescence photon counts as a function of the sweep voltage. For calibration purposes, a measuring sequence usually includes two reference isotopes with well-known IS. The different voltage readings, performed by the computer, constitute the essential information for calculating IS and hfs splittings.

\section{MEASUREMENTS ON UNSTABLE ISOTOPES}

First experiments were performed at the Mainz reactor in $1978^{19-21)}$, yielding IS and hfs for a number of neutron-rich rubidium and caesium isotopes. Recently, the improved apparatus has been connected to ISOLDE, and first results were obtained for barium ${ }^{22}$ ) and ytterbium ${ }^{23)}$.

\subsection{Rubidium and caesium}

The fission-produced isotopes of rubidium and caesium are supplied by an online isotope separator connected to the TRIGA reactor at Mainz ${ }^{24)}$. The output beam has an energy of 5 to $7 \mathrm{keV}$, and yields of the most abundant isotopes ${ }^{90} \mathrm{Rb}$ and ${ }^{138} \mathrm{Cs}$, produced mainly by the decay of primary fission products in the target, lie between $10^{8}$ and $10^{9}$ atoms $/ \mathrm{s}$. The energy spread of the beam, extracted from a surface ionization source at $1200{ }^{\circ} \mathrm{C}$, is about $0.5 \mathrm{eV}$. This corresponds to a Doppler broadening of $12 \mathrm{MHz}$ for the transition $5 \mathrm{~s}^{2} \mathrm{~S}_{1 / 2}-6 \mathrm{p}^{2} \mathrm{P}_{3 / 2}$ in $\mathrm{Rb} \mathrm{I}$ 
$\left(\lambda=4201 \AA\right.$, $\left.\delta \nu_{\text {nat }}=1.4 \mathrm{MHz}\right)$ and $9 \mathrm{MHz}$ for the transition $6 \mathrm{~s}^{2} \mathrm{~S}_{\frac{1}{2}}-7 \mathrm{p}^{2} \mathrm{P}_{3 / 2}$ in Cs I $\left(\lambda=4555 \AA, \delta \nu_{\text {nat }}=1.2 \mathrm{MHz}\right)$.

A spectrum of the six hfs components is shown in fig. 2 for the example of ${ }^{91} \mathrm{Rb}(\mathrm{I}=3 / 2)$. The 1 ine-width of $0.8 \mathrm{~V}$, corresponding to $18 \mathrm{MHz}$ in the frequency scale, includes an additional broadening by the beam divergence. In the observed spectrum, there is a distinct satellite peak to each hfs component, separated from the main peak by $1.6 \mathrm{~V}$. It is due to the energy loss suffered in charge transfer by those of the rubidium atoms which are formed in the first excited $5 p$ state. Since a rubidium vapour target was used for charge exchange, the resonant channel of the reaction is clearly predominant ${ }^{15}$ ). This is important in order that the hfs pattern is not confused by the neutralization energy-loss spectrum.

Since the Doppler width is about 10 times larger than the natural line-width, only about $10 \%$ of the atoms contribute simultaneously to the resonance signal. Under these conditions the sensitivity limit was about $10^{7}$ mass-separated ions per second for isotopes with $I \neq 0$, where weak laser power had to be used to avoid optical pumping. An increase in sensitivity by a factor of 10 could be achieved for the directly fission-produced ${ }^{93} \mathrm{Rb}$ by running the reactor in a pulsed mode. This allows the activity of $120 \mathrm{~s}$ continuous operation to be concentrated within a few $100 \mathrm{~ms}$, with a corresponding reduction of background.

The full series of experiments covers $\mathrm{IS}$ and hfs measurements for ${ }^{89-9{ }^{3} \mathrm{Rb}}{ }^{21}$ ) and $\left.{ }^{138-142} \mathrm{Cs}{ }^{20}\right)$. Studies of these isotopic chains have been continued by the Orsay group at ISOLDE using atomic beam laser optical pumping ${ }^{25}$ ) in the strong infrared resonance lines. Data at present available cover the range of ${ }^{76-98} \mathrm{Rb}{ }^{26}$ ) and ${ }^{118-145} \mathrm{Cs}^{27)}$.

\subsection{Barium}

The ISOLDE yields of barium are between $10^{8}$ and $10^{5}$ atoms/s for the neutronrich isotopes ${ }^{139-146} \mathrm{Ba}$, obtained by $600 \mathrm{MeV}$ proton-induced fission of uranium ${ }^{28}$ ). The neutron-deficient isotopes, produced by spallation in a molten lanthanum target $\left.^{29}\right)$, are generally available at higher intensities of $10^{9}$ to $10^{10}$ atoms $/ \mathrm{s}$ 
around mass number 128 . The yields drop to about $10^{6}$ atoms/s for ${ }^{122} \mathrm{Ba}$, and the odd high-spin isomers between ${ }^{137 m_{B a}}$ and $129 m_{B a}$ are efficiently produced as well. The predominant yield of isobaric caesium is somewhat disturbing because of its radioactivity.

In the $\mathrm{Ba} I$ transition $6 \mathrm{~s}^{2}{ }^{1} \mathrm{~S}_{0}-6 \mathrm{~s} 6 \mathrm{p}{ }^{1} \mathrm{P}_{1}(\lambda=5536 \AA)$, accurate measurements of IS and hfs for all stable and a number of neutron-deficient barium isotopes, as well as some isomers, had been performed by Nowicki et al. ${ }^{30)}$ and Bekk et al. ${ }^{31}$ ). The present on-line work extends to the neutron-rich and very neutron deficient sides, including two more isomers. The compilation of data, now almost complete for $122 \leq \mathrm{A} \leq 146$, is shown in fig. 3 in a frequency scale, relative to the position of the line in ${ }^{138} \mathrm{Ba}$.

Figure 4 shows the example of a measured spectrum for the case of ${ }^{143} \mathrm{Ba}$, with ${ }^{137} \mathrm{Ba}$ and ${ }^{134} \mathrm{Ba}$ as the stable reference isotopes. From the acceleration voltages, the IS and hfs frequency intervals are calculated by

$$
v_{2}-v_{1}=v_{0}\left\{\left(\frac{2 \mathrm{eU}_{1}}{\mathrm{~m}_{1} \mathrm{c}^{2}}\right)^{\frac{1}{2}}-\left(\frac{2 \mathrm{eU}_{2}}{\mathrm{~m}_{2} \mathrm{c}^{2}}\right)^{\frac{1}{2}}\right\} .
$$

(For the sake of clarity, higher-order terms in the Doppler shift have been omitted.) The atomic masses are accurately known in most cases ${ }^{31)}$. For a few isotopes, one has to rely on mass calculations which are believed to be accurate within $10^{-5}$, thus contributing slightly to the errors of calculated IS. Otherwise, systematic errors are mainly due to the voltage measurements, which are accurate within $10^{-4}$. Including statistical errors and uncertainties in the analysis of the line shape, one ends up with final errors between 1 and $10 \mathrm{MHz}$, with the general trend that small frequency intervals are more accurately measured than large ones. An on-line test of this accuracy is provided by the reference signals for which the frequency differences are known ${ }^{30}$ ).

The observed line-width is about $35 \mathrm{MHz}$, to be compared with a natural linewidth of $19 \mathrm{MHz}$. The residual Doppler broadening can be attributed to the ion source, the beam divergence and inelastic channels of the charge transfer reaction. These different contributions will be subject to further studies. 
An estimate of the experimental sensitivity can be performed on the basis of the weak signals of ${ }^{146} \mathrm{Ba}$, obtained within $15 \mathrm{~min}$ from a beam of $10^{5}$ atoms/s with a signa1-to-noise ratio of 10 . The photon collection efficiency of $10^{-2}$ photons/ atom is consistent with the estimate of Section 3.1, if 100 excitations per atom with some loss due to non-quantitative charge transfer, non-perfect overlap of the beams, and residual Doppler broadening are assumed. An increase of the measuring time to the order of an hour is possible, limitations arising from mode hops of the laser system and the procedure of localizing the resonances. With perfect stray-light conditions, a sensitivity limit can thus be established for even Ba isotopes at about $10^{4}$ atoms/s. For odd isotopes this limit is more than a factor of 10 higher, since optical pumping by multiple excitation has to be avoided, and the sensitivity is determined by the weakest hfs component in the spectrum.

\subsection{Ytterbium}

For ytterbium, the intercombination line $6 \mathrm{~s}^{2}{ }^{1} \mathrm{~S}_{0}-6 \mathrm{~s} 6 \mathrm{p}^{3} \mathrm{P}_{1}(\lambda=5556 \AA)$ is easily accessible, but the long lifetime of the ${ }^{3} \mathrm{P}_{1}$ state, $\left.\tau=827(40) \mathrm{ns}^{33}\right)$, implies some disadvantages. During this time, an ytterbium atom in the $60 \mathrm{keV}$ beam travels more than $20 \mathrm{~cm}$, emitting on the average less than one spontaneous photon in the observation region. Furthermore, the natural line-width of $200 \mathrm{kHz}$ is small compared with the absorption Doppler width of about $20 \mathrm{MHz}$. Consequently, about $1 \%$ of the atoms in the beam can absorb the narrow-band laser radiation. This efficiency can be increased by power broadening, but a higher background from scattered laser light has to be accepted. Including this gain, the experimental detection limit was found to be some $10^{7}$ atoms $/ \mathrm{s}$.

The ytterbium beams are produced by the spallation of tantalum and surface ionization in a tungsten tube at $2300{ }^{\circ} \mathrm{C}{ }^{34}$ ). Yields of up to $10^{9}$ atoms/s can be achieved at maximum around $A=166$. In a first attempt with much weaker beams, the IS of the doubly-even radioactive isotopes ${ }^{166} \mathrm{Yb},{ }^{164} \mathrm{Yb}$, and ${ }^{162} \mathrm{Yb}$ has been measured, relative to the lightest stable isotope ${ }^{168} \mathrm{Yb}$, connecting them to the series of precision measurements made by Clark et al. ${ }^{35}$ ) on stable isotopes. 


\section{EVALUATION OF NUCLEAR DATA}

Nuclear spins, magnetic dipole moments, and electric quadrupole moments have to be deduced from the measured hfs splittings. The IS carry information about the change of mean-square nuclear charge radii. These calculations require knowledge of electron-shel1 parameters entering into the hyperfine interaction.

\subsection{Hyperfine structure}

The hyperfine splitting of a spectral line reflects the splitting of the states involved in the transition. Within one state, it can be described by

$$
\mathrm{W}_{\mathrm{F}}=\frac{1}{2} \mathrm{KA}+\frac{3 / 4 \mathrm{~K}(\mathrm{~K}+1)-\mathrm{I}(\mathrm{I}+1) \mathrm{J}(\mathrm{J}+1)}{2 \mathrm{I}(2 \mathrm{I}-1) \mathrm{J}(2 \mathrm{~J}-1)} \mathrm{B} \text {, }
$$

with

$$
K=F(F+1)-I(I+1)-J(J+1),
$$

where $I$ is the nuclear spin, $J$ is the angular momentum of the shell electrons, and $\mathrm{F}$ is the total angular momentum for the different hfs states

$(|\mathrm{J}-\mathrm{I}| \leq \mathrm{F} \leq|\mathrm{J}+\mathrm{I}|)$

The magnetic dipole interaction constant

$$
A=\frac{\mu_{I}\left\langle H_{e}(0)\right\rangle}{I J}
$$

and the electric quadrupole interaction constant

$$
B=Q_{s}\left\langle\frac{\partial^{2} V}{\partial z^{2}}\right\rangle
$$

can be separated into an electronic factor and the magnetic moment $\mu_{I}$ or the spectroscopic quadrupole moment $Q_{S}$, respectively. The magnetic field of the electrons at the nuclear site $\left\langle\mathrm{H}_{\mathrm{e}}(0)\right\rangle$ is known from the hfs and directly measured nuclear g-factors in stable isotopes. If it is used to calculate nuclear moments from the hfs in other isotopes, an error accounting for the hyperfine anomaly ${ }^{36}$ ) has to be introduced. The electric field gradient at the nuclear site $\left\langle\partial^{2} \mathrm{~V} / \partial z^{2}\right\rangle$ is not accessible to direct measurement, but has to be calculated from electron 


\section{$-11-$}

wave functions ${ }^{37)}$. The problem of large uncertainties in these calculations is going to be solved by the increasing number of $Q_{S}$ measurements in muonic atoms ${ }^{38)}$. According to eq. (4) the nuclear spin can be determined if more than three hfs levels are observed. Since this is not the case for ${ }^{1} \mathrm{~S}_{0}-{ }^{1},{ }^{3} \mathrm{P}_{1}$ transitions (e.g. $\mathrm{Ba}$ and $\mathrm{Yb}$ ), the spins have to be known from elsewhere. For the odd $\mathrm{Ba}$ isotopes, the spin has been deduced from the intensity ratios of the hfs components which are governed by Clebsch-Gordan coefficients.

\subsection{Isotope shift}

The centre of gravity of an optical hfs multiplet is sensitive to the radial distribution of the nuclear charge. Its shift between two isotopes is the sum of the so-called field shift, caused by a change of the charge distribution, and the mass shift, caused by the change of the nuclear recoil energy ${ }^{39}$ )

$$
\delta \nu^{A A^{\prime}}=\delta \nu_{f}^{A A^{\prime}}+M \frac{A^{\prime}-A}{A^{\prime} A}
$$

The mass-shift constant $M$ is of the order $\nu / 1836$, which accounts for the reduced mass in a one-electron atom (normal mass shift). Corrections due to electron correlation (specific mass shift) happen to be small for s-p transitions which, on the other hand, have large field shifts. Theoretical estimates and the comparison with experimental results from muonic and electronic X-ray spectra show that $M=(\nu / 1836)(1 \pm 1)$ is a reasonable assumption.

The field shift is related to the difference of mean-square nuclear charge radii $\delta\left\langle\mathrm{r}^{2}\right\rangle^{\mathrm{AA}}$ by

$$
\delta \nu_{f}^{\mathrm{AA}_{\mathrm{f}}^{\prime}}=\frac{2}{3} \cdot \pi Z \mathrm{e}^{2} \Delta|\psi(0)|^{2} \delta\left\langle\mathrm{r}^{2}\right\rangle^{\mathrm{AA}^{\prime}}
$$

where $\Delta|\psi(0)|^{2}$ is the change of the electron density at the nucleus in the electronic transition. For high $Z$ this non-relativistic equation has to be modified to take into account relativistic corrections and the contribution of higher radial moments of the charge distribution. An empirical value of $\Delta|\psi(0)|^{2}$ for s-p transitions in alkali-like spectra can be based on the Fermi-Goudsmit-Segrè 
formula for the fine structure energies, or the contact term of the hyperfine interaction, both yielding $|\psi(0)|^{2}$ of the s-state involved. For $\mathrm{s}^{2}$-sp transitions, this quantity can be determined in the ionic spectrum and changed by an empirical screening ratio accounting for the second s electron.

Whenever possible, the electronic factor of the field shift as well as the specific mass-shift constant should be calibrated by comparison with IS data from $\mathrm{X}$-ray and muonic spectra, for which accurate calculations can be made ${ }^{40)}$. This information, however, needs high-precision experiments and can only be complete for elements with at least three stable isotopes.

\section{RESULTS AND THEIR IMPACT ON NUCLEAR PHYSICS}

Ground-state spins are among the key information in nuclear structure research. Their direct measurement by atomic spectroscopy forms the basis of spin assignments in nuclear spectroscopy. The spins alone may in many cases identify the main contribution to the nuclear wave function in terms of she11-model or Nilsson-model states. A qualitative indication of nuclear deformation is thus also obtained from the spins in a sequence of isotopes. Systematic spin measurements are carried out at ISOLDE with an on-1ine variant of Rabi's atomic beam magnetic resonance method ${ }^{41)}$. They are complemented by the information from optical spectroscopy, which holds a monopoly of spin measurements for the elements with diamagnetic atomic ground states.

Magnetic moments are also measured by radio-frequency spectroscopy of atomic ground states (see ref. 41 and refs. 6-9) or hyperfine spectroscopy in optical lines. They give more accurate information on the nuclear state assignments, and remove ambiguities in the interpretation of spins.

The measurement of quadrupole moments is usually the domain of optical spectroscopy, since many elements that are easily accessible to on-line isotope separation have $J=0$ or $J=\frac{1}{2}$ atomic ground states. Optical transitions, however, can be chosen to involve excited states with $J \geq 1$. One of the merits of laser spectroscopy is its resolution of the small quadrupole interaction in most of the 
elements. The interpretation of the experimental spectroscopic quadrupole moment is somewhat ambiguous because of the model dependence of the projection factor relating it to the intrinsic quadrupole moment. However, some transitional nuclei have recently been successfully treated within the core-quasiparticle coupling model of Larsson et a1. ${ }^{42)}$.

A more direct access to nuclear deformation is provided by the IS measurements. Once the variation of mean-square charge radii in a sequence of isotopes is evaluated according to eq. (6), it can be separated into two parts, the one corresponding to a change of nuclear size at constant shape, the other corresponding to a change of deformation:

$$
\delta\left\langle\mathrm{r}^{2}\right\rangle^{\mathrm{AA}^{\prime}}=(2 / 5) \rho \mathrm{R}_{0}^{2} \frac{\mathrm{A}^{\prime}-\mathrm{A}}{\frac{1}{2}\left(\mathrm{~A}^{\prime}+\mathrm{A}\right)}+\frac{3}{4 \pi} \mathrm{R}_{0}^{2} \delta\left\langle\beta^{2}\right\rangle
$$

Here the first term represents the so-called standard IS describing the expansion of a uniformly charged liquid drop of radius $R_{0}$. It is multiplied by an empirical factor $\rho \approx 0.5$, which fits the over-all trend of IS and has also some theoretical support from the droplet mode1 $\left.{ }^{43}\right)$. Assuming this simple two-parameter description, we can follow the change of deformation along an isotopic chain. Absolute values of $\left\langle\beta^{2}\right\rangle$ are obtained if this quantity is known from $B(E 2)$ reduced transition probabilities for at least one isotope. At neutron-shell closures the deformation is sma11, and $\left\langle\beta^{2}\right\rangle$ can be assumed to be zero if no further information is available.

For illustration, the reduced standard value with $\rho=0.5$ is plotted in fig. 5, together with the experimental $\delta\left\langle\mathrm{r}^{2}\right\rangle$ values obtained around $\mathrm{N}=82$. The deviation of the experimental lines from the reduced standard line is thus attributed to the change of deformation represented by the second term in eq. (7). To give an idea of the corresponding deformation parameters, 1ines of constant $\left\langle\beta^{2}\right\rangle$ are shown with the simplification $\left\langle\beta^{2}\right\rangle=0$ for $N=82$. The exact value, $\left\langle\beta^{2}\right\rangle=$ $=0.0084$, is known only for ${ }^{138} \mathrm{Ba}^{44)}$. In this picture, the characteristic kink of the experimental lines at $\mathrm{N}=82$ is the natural consequence of deformation, 
increasing with distance from the magic neutron number. The increasing distance from the closed proton shell at $\mathrm{Z}=50$ in the sequence ${ }_{54} \mathrm{Xe},{ }_{5} \mathrm{Cs},{ }_{6} \mathrm{Ba}$ would offer an obvious explanation of the $Z$ dependence of the effect. There is, however, the uncertainty of specific mass shifts and electronic factors (see Section 5.2) which complicates the comparison of data for different elements.

More complete information on the neighbouring elements, improved calculations of $\delta\left\langle r^{2}\right\rangle$ on the basis of calibration measurements in muonic atoms, and a few more absolute values of $\left\langle\beta^{2}\right\rangle$ from $B(E 2)$ measurements are needed to establish the situation quantitatively. In fact, caesium and barium are the first examples of precise IS measurements in long isotopic chains of neighbouring elements ranging to both sides of closed neutron shell.

7. CONCLUSION

The various approaches to on-line application of hyperfine spectroscopy, using the minute samples of radioactive atoms, have revealed fiew, relevant, and otherwise inaccessible information on nuclear structure. Among these methods, collinear laser spectroscopy has proved to be of most general use, yielding at the same time the maximum possible information. Practical limits arise from the accessibility of resonance transitions to present CW dye lasers. Here, the promising attempts of intracavity frequency-doubling in ring lasers may open up the UV. On the other hand, selective charge transfer to metastable states could be used for elements with low-lying ground states, such as the rare gases.

The sensitivity of the method will mainly depend on the wavelength of the transition, the lifetime of the excited state involved, and the complexity of the spectrum. In some cases, it will be helpful to observe the decay to a third state $^{45)}$ in order to get rid of the stray light background. The relatively low efficiency of optical detection might be overcome by stepwise laser excitation, leading to ionization and subsequent particle counting ${ }^{46}$ ).

The present sensitivity limit has been established to be some $10^{4}$ atoms/s for the even Ba isotopes. This may be improved in favourable cases. For a 
majority of elements, however, higher-intensity beams will be needed, but the yields of on-line isotope separators may still be sufficient for comprehensive work. For any particular case, the experiments will require new ideas and specific developments in order that the method may be adapted to the experimental situation of rather differently behaving elements. 


\section{REFERENCES}

1) P.G. Hansen, Annu. Rev. Nuc1. Part. Sci. 29 (1979) 69.

2) B. Jonson, Trends in the study of nuclei by means of on-line separators, Contribution to these Proceedings, to be published in Nucl. Instrum. Methods.

3) H.L. Ravn, Phys. Reports 54 (1979) 201.

4) H. Kopfermann, Nuclear moments (Academic Press, Inc., New York, 1958).

5) K. Shimoda (ed.), High-resolution laser spectroscopy in Topics in Applied Physics, Vol. 13 (Springer, Berlin-Heidelberg-New York, 1976).

6) E.W. Otten, Proc. Int. Conf. on the Properties of Nuclei far from the Region of Beta-Stability, Leysin, 1970 (CERN 70-30, Geneva, 1970), Vo1. 1, p. 361.

7) E.W. Otten, Proc. 5th Int. Conf. on Atomic Physics, Berkeley, 1976 (Plenum Pub1. Corp., New York, 1977), p. 239.

8) H.-J. Kluge, in Progress in Atomic Spectroscopy (eds. W. Hanle and H. Kleinpoppen) (Plenum Pub1. Corp., New York and London, 1979), Part B, p. 727 .

9) P. Jacquinot and R. Klapisch, Rep. Progr. Phys. 42 (1979) 773.

10) E.W. Otten, Laser techniques in nuclear physics, Proc. Int. Conf. on Nuclear Physics, Berkeley, 1980, to be published.

11) R. Klapisch, Nuclear properties studies by atomic physics, Proc. 7 th Int. Conf. on Atomic Physics, Cambridge, Mass., 1980, to be published.

12) R. Neugart, Proc. 5th Int. Conf. on Beam-Foil Spectroscopy, Lyon, 1978, J. Phys. 40 (1979) C1-38.

13) H.S.W. Massey and H. Gilbody, Electronic and Ionic Impact Phenomena (C1arendon Press, Oxford, 1974), Vo1. 4.

14) S.L. Kaufman, Opt. Commun. 17 (1976) 309. 
15) K.-R. Anton, S.L. Kaufman, W. Klempt, G. Moruzzi, R. Neugart, E.W. Otten and B. Schinzler, Phys. Rev. Lett. 40 (1978) 642.

16) W.H. Wing, G.A. Ruff, W.E. Lamb, Jr. and J.J. Spezeski, Phys. Rev. Lett. $\underline{36}$ (1976) 1488 .

17) Th. Meier, H. Hühnermann and H. Wagner, Opt. Commun. 20 (1977) 397.

18) K.-H. Georgi and B. Vošicki, private communication.

K.-H. Georgi, Contribution to these Proceedings, High-voltage stabilization for an on-line isotope separator, to be published in Nucl. Instrum. Methods.

19) B. Schinzler, W. Klempt, S.L. Kaufman, H. Lochmann, G. Moruzzi, R. Neugart, E.W. Otten, J. Bonn, L. von Reisky, K.P.C. Spath, J. Steinacher and D. Weskott, Phys. Lett. 79B (1978) 209.

20) J. Bonn, W. Klempt, R. Neugart, E.W. Otten and B. Schinzler, Z. Phys. A289 (1979) 227.

21) W. Klempt, J. Bonn and R. Neugart, Phys. Lett. 82B (1979) 47.

22) R. Neugart, F. Buchinger, W. Klempt, A.C. Mueller, E.W. Otten, C. Ekström and J. Heinemeier, Fast-beam laser spectroscopy of neutron-rich barium isotopes, Proc. 5th Int. Conf. on Hyperfine Interactions, Berlin, 1980, to be published in Hyperfine Interactions.

23) F. Buchinger, W. Klempt, A.C. Mueller, E.W. Otten, C. Ekström and R. Neugart, Isotope shift of ${ }^{162} \mathrm{Yb},{ }^{164} \mathrm{Yb}$, and ${ }^{166} \mathrm{Yb}$ determined by collinear-beam laser spectroscopy, Contribution to the 12th EGAS Conf., Pisa, 1980.

24) L. von Reisky, J. Bonn, S.L. Kaufman, L. Kugler, E.W. Otten, J. Rodriguez-Giles, K.P.C. Spath and D. Weskott, Nuc1. Instrum. Methods 172 (1980) 423.

25) G. Huber, C. Thibault, R. Klapisch, H.T. Duong, J.L. Vialle, J. Pinard, P. Juncar and P. Jacquinot, Phys. Rev. Lett. 34 (1975) 1209.

26) C. Thibault, F. Touchard, S. Büttgenbach, R. Klapisch, M. de Saint Simon, H.T. Duong, P. Jacquinot, P. Juncar, S. Libermann, P. Pillet, J. Pinard, J.L. Vialle, A. Pesnelle and G. Huber, Hyperfine structure and isotope shift of the $\mathrm{D}_{2}$ line of ${ }^{76-98} \mathrm{Rb}$ and some of their isomers, to be published in Phys. Rev. C. 
27) Private communication by authors of ref. 26. See also ref. 11 .

28) L.C. Carraz, H.L. Ravn, M. Skarestad, S. Sunde11 and L. Westgaard, Nucl. Instrum. Methods 158 (1979) 69.

29) H.L. Ravn, S. Sunde11 and L. Westgaard, Nuc1. Instrum. Methods $\underline{123}$ (1975) 131 .

30) G. Nowicki, K. Bekk, S. Göring, A. Hanser, H. Rebel and G. Schatz, Phys. Rev. C 18 (1978) 2369 .

31) K. Bekk, A. Andl, S. Göring, A. Hanser, G. Nowicki, H. Rebel and G. Schatz, Z. Phys. A291 (1979) 219.

32) A.H. Wapstra and K. Bos, Atomic Data and Nuclear Data Tables 19 (1977) 177.

33) M. Baumann and G. Wande1, Phys. Lett. 22 (1966) 283.

34) H.L. Ravn, L.C. Carraz, J. Denimal, E. Kugler, M. Skarestad, S. Sunde11 and L. Westgaard, Nuc1. Instrum. Methods $\underline{139}$ (1976) 267.

35) D.L. Clark, M.E. Cage, D.A. Lewis and G.W. Greenlees, Phys. Rev. A 20 (1979) 239.

36) G.H. Fuller and V.W. Cohen, ORNL-4591 (1970).

37) I. Lindgren and A. Rosén, Case Studies in Atomic Physics $\underline{4}$ (1974) 197.

38) R.M. Steffen, Proc. 5th Int. Conf. on Hyperfine Interactions, Berlin (West), 1980, to be published in Hyperfine Interactions.

39) K. Heilig and A. Steudel, Atomic Data and Nuclear Data Tables 14 (1974) 613.

40) See, for example, the compilations of R. Engfer, H. Schneuwly, J.L. Vuilleumier, H.K. Walter and A. Zehnder, Atomic Data and Nuclear Data Tables 14 (1974) 509 .

F. Boehm and P.L. Lee, Atomic Data and Nucl. Data Tables 14 (1974) 605 .

41) C. Ekström, Application of on-line ABMR techniques to nuclides far from stability, Contribution to these Proceedings, to be published in Nucl. Instrum. Methods. 
42) S.E. Larsson, G. Leander and I. Ragnarsson, Nucl. Phys. A307 (1978) 189.

43) W.D. Myers, Phys. Lett. 30B (1969) 451.

44) A. Christy and O. Häusser, Nucl. Data Tables 11 (1972) 281.

45) H. Wagner, K. Dörschel, C. Höhle, H. Hühnermann and Th. Meier, Laser fluorescence spectroscopy on fast ion beams, Contribution to these Proceedings, to be published in Nucl. Instrum. Methods.

46) V.S. Letokhov, Proc. 4th Int. Conf. on Laser Spectroscopy, Rottach-Egern, 1979, Springer Series in Optical Sciences (Springer, Berlin-HeidelbergNew York, 1979), Vo1. 21, p. 504. 


\section{Figure captions}

Fig. 1 : Set-up for on-line collinear-beam laser spectroscopy.

Fig. 2 : hfs level scheme and corresponding resonance pattern observed for ${ }^{91} \mathrm{Rb} \quad($ ref. 21).

Fig. 3 : Positions of hfs components and their centres of gravity in the Ba $I$ line $5536 \AA$ for the isotopes ${ }^{122-146} \mathrm{Ba}$ and some of their isomers, with respect to ${ }^{138} \mathrm{Ba}$. The figure includes data of Nowicki et al. ${ }^{30}$ ), and Bekk et $\left.a 1 .{ }^{31}\right)$, and results recently obtained at $\operatorname{ISOLDE}^{22}$ ). The latter are marked by arrows, and the spin assignments are given on top.

Fig. 4 : Spectrum of ${ }^{143} \mathrm{Ba}$ with simultaneously measured reference spectra ${ }^{22}$ ). Nuclear spins are obtained by comparing the relative intensities of hfs components.

Fig. 5 : Plot of $\delta\left\langle\mathrm{r}^{2}\right\rangle$ for the series ${ }^{122-146} \mathrm{Ba}{ }^{30,31,22)}, 118-145 \mathrm{Cs}{ }^{27)}$, and $124-136 \mathrm{Xe}^{39)}$. The assumption $\rho=0.5$ and $\left\langle\beta^{2}\right\rangle^{\frac{1}{2}}=0$ at $\mathrm{N}=82$ is made to show lines of constant r.m.s. deformation. Isomers are only included in cases of large shifts. 


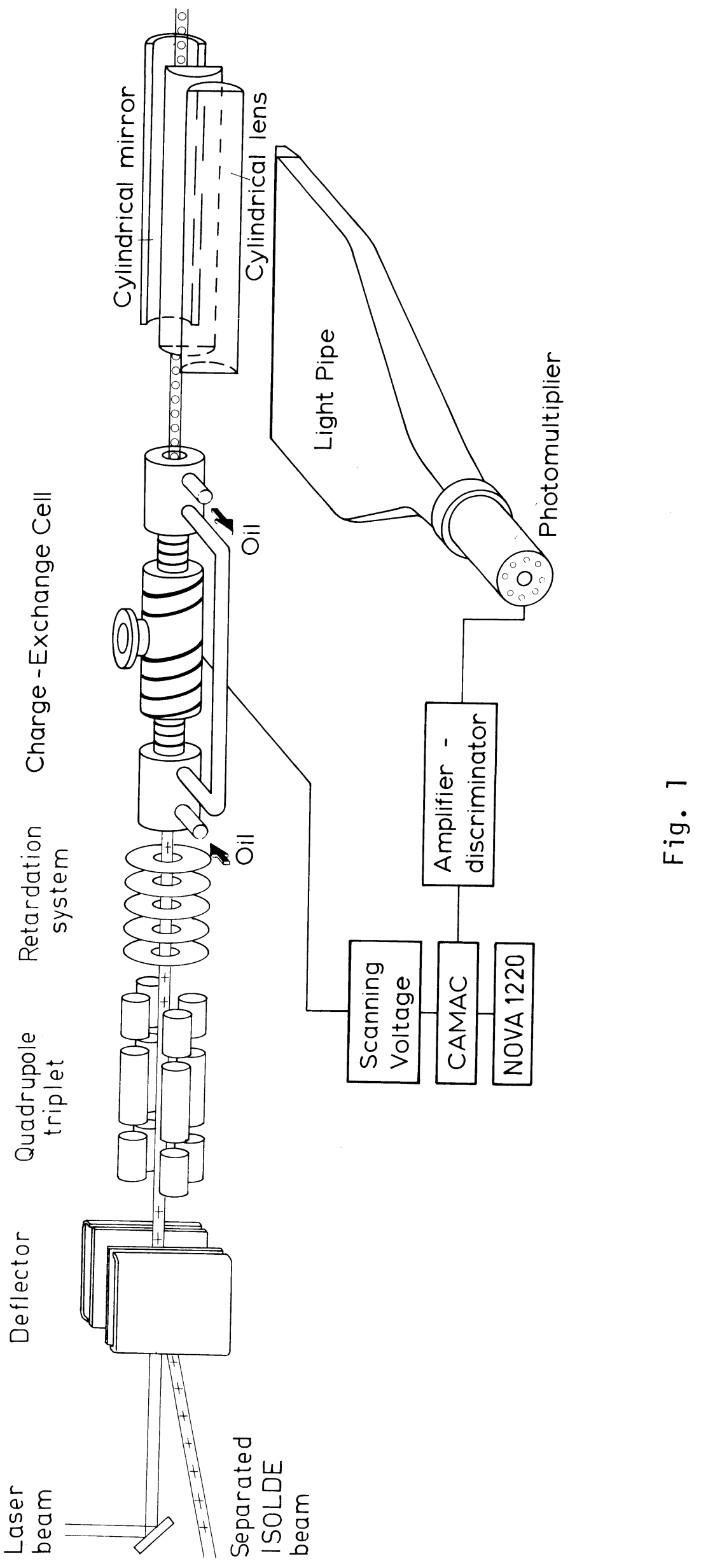




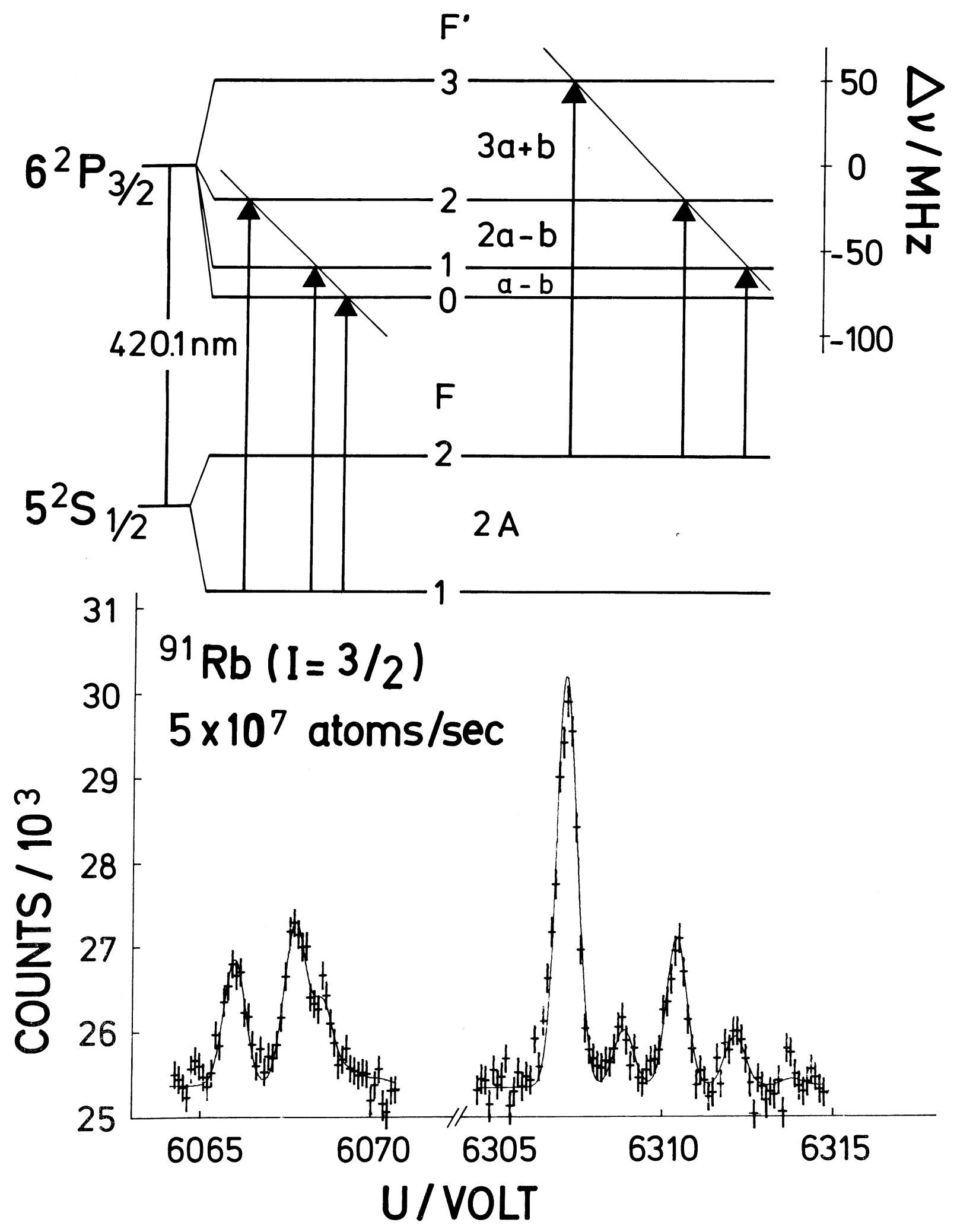

Fig. 2 


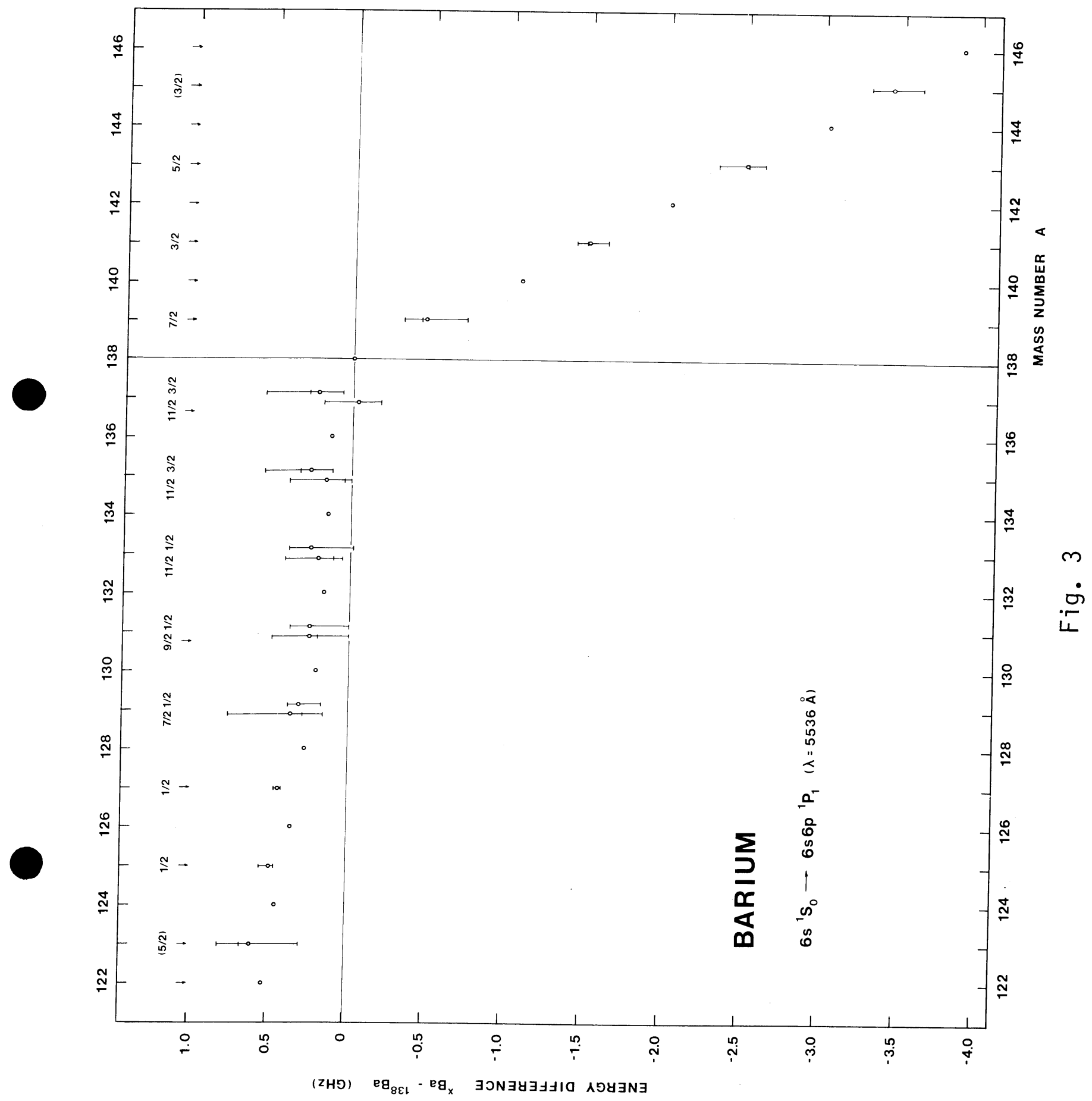




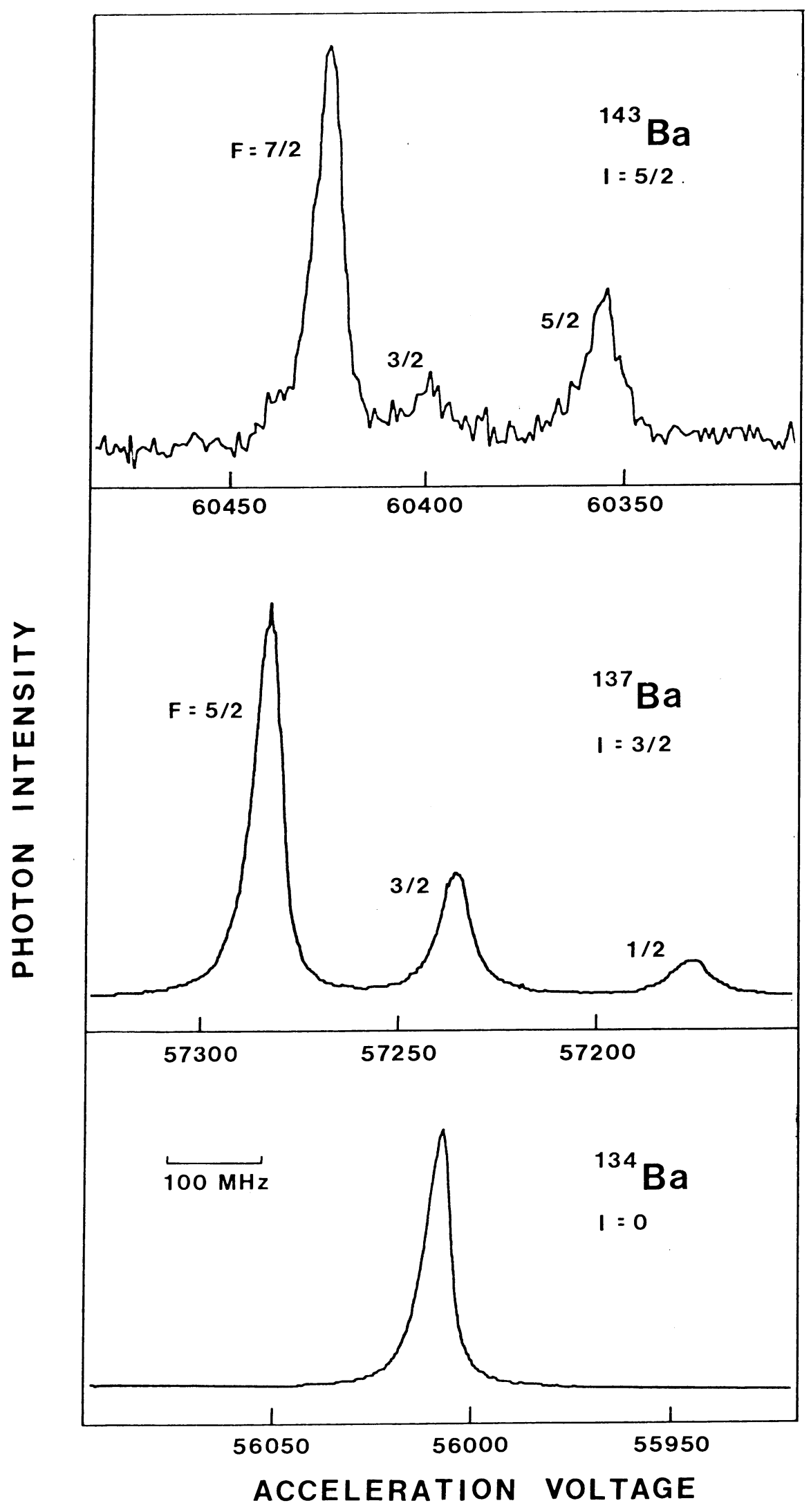

Fig. 4 


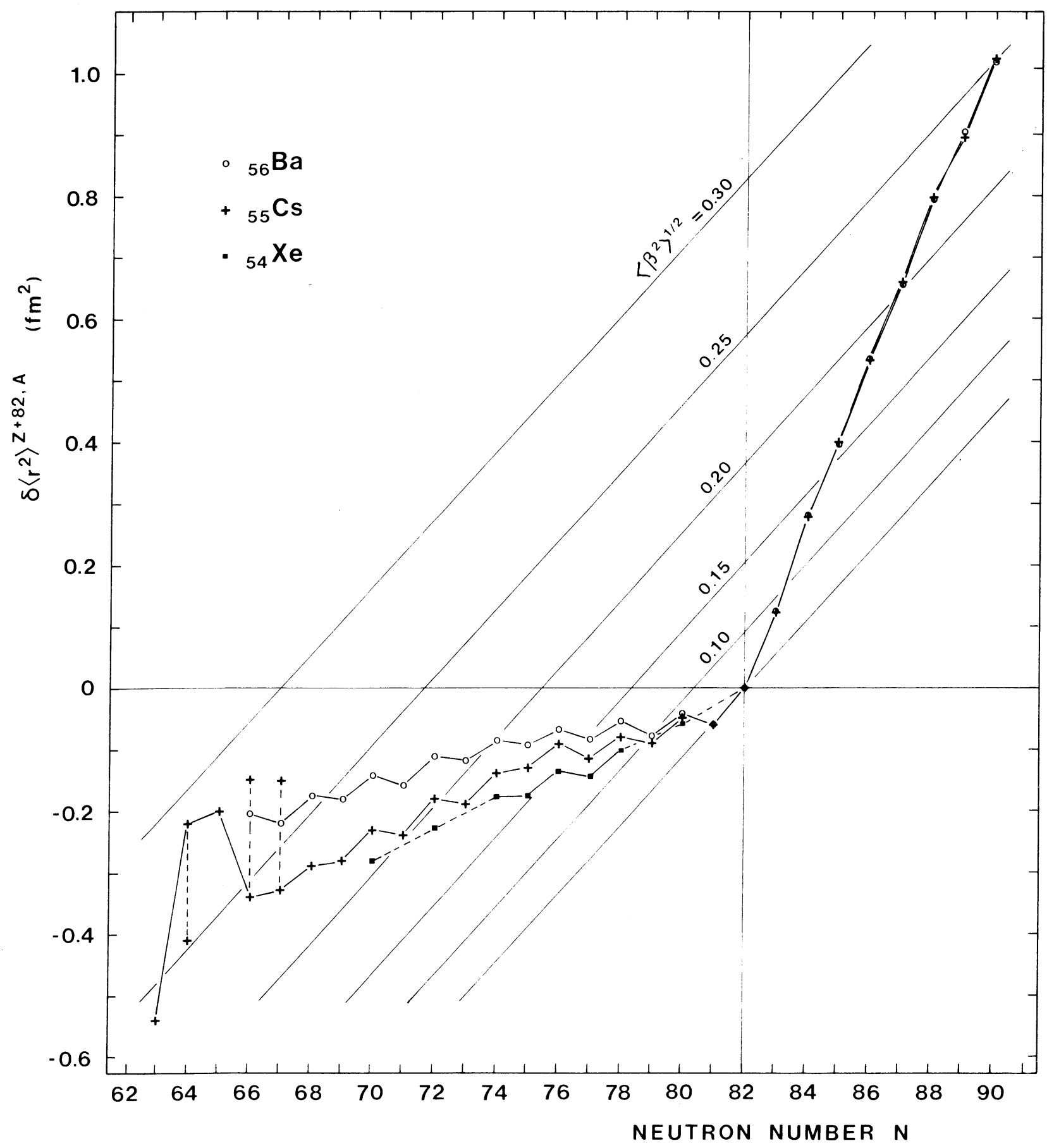

Fig. 5 